\section{Deutsche Krebsgesellschaft e.V.}

Deutsche Krebsgesellschaft e.V.

Hanauer Landstr. 194, D-60314 Frankfurt

Tel. +49 696300 96-0, Fax -66

E-Mail service@krebsgesellschaft.de

Internet http://www.krebsgesellschaft.de

Für Sicherheit in Diagnose und Therapie von Brustkrebs

\section{Erste evidenzbasierte Leitlinie zur Brustkrebs-Behandlung} im Netz

Die Deutsche Krebsgesellschaft hat gemeinsam mit anderen medizinischen Fachgesellschaften eine evidenzbasierte Leitlinie «Diagnostik und Therapie des Mammakarzinoms der Frau» erarbeitet. Diese Leitlinie ist auf nationaler Ebene die erste Handlungsempfehlung, die die medizinische Versorgung von an Brustkrebs erkrankten Frauen mit größtmöglicher wissenschaftlicher Absicherung festlegt. Bereits existierende evidenzbasierte Leitlinien anderer Länder sowie anerkannte internationale Kriterien wurden in die nationale Leitlinie eingearbeitet. Patientinnen waren mit ihren Selbsthilfeorganisationen in die Entscheidungsprozesse eingebunden.

Nach einer aufwändigen Erarbeitungszeit von vielen Monaten liegt nun die vorläufige Endfassung der so genannten Stufe 3 Leitlinie vor. Unter www.krebsgesellschaft.de im Channel DKG - Wir über uns / ISTO / Evidenzbasierte Leitlinien kann sie ab sofort eingesehen und abgerufen werden. Die vorläufige Endfassung muss nach Bewertung der wissenschaftlichen Studienlage durch ein abschließendes Konsensusverfahren verabschiedet werden. Die Deutsche Krebsgesellschaft weist daher ausdrücklich darauf hin, dass Änderungen nicht ausgeschlossen sind. Um jedoch größtmögliche Transparenz zu schaffen und über die voraussichtlichen Inhalte zu informieren, hat sich die Deutsche Krebsgesellschaft entschlossen, die Leitlinie vorab online zu veröffentlichen.

In der Bundesrepublik Deutschland erkranken jährlich etwa 46000 Frauen an Brustkrebs. Brustkrebs ist damit die häufigste Krebserkrankung der Frau. Durch gezielte Maßnahmen der Qualitätssicherung und Bündelung von Kompetenzen kann eine hochwertige medizinische Versorgung gewährleistet werden. Die jetzt vorgelegte nationale Leitlinie soll den Ärzten in Brustzentren und deren Umfeld helfen, die Diagnostik und Behandlung von Patientinnen mit Brustkrebs zu optimieren. Sie ist die entscheidende Grundlage für das bundesweit anlaufende Disease-Management-Programm oder Chronikerprogramm zum Brustkrebs.

Die Leitlinie der Deutschen Krebsgesellschaft hat Vorbildfunktion für zukünftig zu erstellende S3 Leitlinien der Krebsmedizin. Gemeinsam mit dem Ärztlichen Zentrum für Qualität in der Medizin (ÄZQ, www.aezq.de) und der Arbeitsgemeinschaft der Wissenschaftlichen Medizinischen Fachgesellschaften (AWMF, www.awmf-online.de) konnte der Prozess für die Erarbeitung derartiger Leitlinien definiert werden.

\section{Deutscher Krebspreis für herausragende Forschungs- arbeiten der Krebsmedizin}

\section{Preisverleihung im Rahmen des 12. Internationalen AEK Krebskongresses}

Die Deutsche Krebsgesellschaft hat in diesem Jahr drei Wissenschaftler mit dem Deutschen Krebspreis ausgezeichnet. Neben Arbeiten der experimentellen Grundlagenforschung sowie der klinischen Tumordiagnostik und -therapie wurden erstmals auch wissenschaftliche Ergebnisse der Transferforschung honoriert. Mit diesem neuen Translationspreis soll die Übertragung von Ergebnissen der Grundlagenforschung hin zur klinischen Anwendung als besonders bedeutsam hervorgehoben werden.

Auf dem 12. Internationalen AEK Krebskongress wurde am 26.3.2003 in Würzburg der Deutsche Krebspreis 2003 in seinem experimentellen, klinischen und translationalen Teil verliehen. Preisträger Prof. Dr. Alfred Wittinghofer, Max-PlanckInstitut für Molekulare Physiologie Dortmund, erhielt die Auszeichnung für seine experimentelle Krebsforschung auf dem Gebiet der G-Proteine der Ras-Familie. Wie durch Mutationen veränderte Ras-Proteine zu Auslösern von Krebs werden und wie man sie in Zukunft vielleicht therapeutisch wieder unter Kontrolle bringen kann, das berichtete Prof. Wittinghofer den interessierten Zuhörern im Würzburger Kongresszentrum.

Prof. Dr. rer. nat. Wolfgang Schlegel, Heidelberg, wurde mit dem diesjährigen klinischen Krebspreis geehrt. Er gilt als geistiger Vater bahnbrechender Entwicklungen im Bereich der dreidimensionalen Bildverarbeitung und Bestrahlungsplanung. Diese Entwicklungen der letzten 15 bis 20 Jahre, die mittlerweile Eingang in die klinische Behandlungspraxis gefunden haben, leistete die Arbeitsgruppe «Radiologische Diagnostik und Therapie» am Deutschen Krebsforschungszentrum mit Prof. Schlegel als Leiter der Forschungsabteilung. 
Prof. Dr. med. Reinhard Dummer, Universitätsspital Zürich, wurde als erster Preisträger mit dem Translationspreis ausgezeichnet. In seinen Forschungsarbeiten zum T-Zell-Lymphom der Haut widmete er sich insbesondere der Analyse der veränderten T-Zellen und damit den Möglichkeiten der Behandlung dieser bösartigen Neubildung. Prof. Dummer verfolgte die Forschung auf diesem Gebiet von der immunbiologischen Basis bis hin zu klinischen Studien. Auch die Immunbiologie des malignen Melanoms der Haut und hier ebenfalls Behandlungsmöglichkeiten wie Impfstrategien zählen zu Prof. Dummers Forschungsschwerpunkten.

Der Deutsche Krebspreis wird jedes Jahr auf dem Deutschen Krebskongress und dem alternierend veranstalteten AEK Krebskongress verliehen. Stifter sind Baxter Oncology $\mathrm{GmbH}$, Pfizer GmbH und Pharmacia Corp. 